\title{
Prospective randomized study of two laparotomy incisions for gastrectomy: midline incision versus transverse incision
}

\author{
Tsuyoshi Inaba, Kota Okinaga, Ryoji Fukushima, Hisae Iinuma, Takashi Ogihara, Fujio Ogawa, \\ Kota Iwasaki, Masanao Tanaka, and Hideki Yamada \\ Department of Surgery, Teikyo University Hospital, 2-11-1 Kaga, Itabashi-ku, Tokyo 173-8605, Japan
}

\begin{abstract}
Background. We performed a randomized study to evaluate the differences between upper midline incision and transverse incision for gastrectomy.

Methods. Patients undergoing distal gastrectomy or total gastrectomy for gastric cancer were randomly allocated to have either an upper midline incision or a transverse incision. The times taken to open and close the abdominal cavity, the number of doses of postoperative analgesics, and the incidence of postoperative pneumonia, wound infection, and intestinal obstruction were compared between the patients having the two incisions.

Results. Times for both opening and closing the abdominal cavity were longer with a transverse incision, in both the distal gastrectomy group and total gastrectomy group. In the patients in whom continuous epidural analgesia was used postoperatively, the number of additional doses of analgesics was smaller in the transverse-incision group after distal gastrectomy. The incidence of postoperative pneumonia was lower in the transverse-incision group after distal gastrectomy. The number of patients with postoperative intestinal obstruction was smaller in the transverse-incision group than in the midline-incision group after distal gastrectomy. In contrast to distal gastrectomy, there was no significant difference in the number of doses of postoperative analgesics, incidence of postoperative pneumonia, or incidence of postoperative intestinal obstruction between the two study groups after total gastrectomy.

Conclusion. A transverse incision for distal gastrectomy may be more beneficial than an upper midline incision in attenuating postoperative wound pain, decreasing the incidence of postoperative pneumonia, and preventing postoperative intestinal obstruction.
\end{abstract}

Key words Midline incision - Transverse incision - Gastrectomy $\cdot$ Gastric cancer $\cdot$ Intestinal obstruction

Offprint requests to: $\mathrm{T}$. Inaba

Received: April 14, 2004 / Accepted: July 5, 2004

\section{Introduction}

Gastrectomy is one of the most common major surgical procedures in gastrointestinal surgery. In Japan, many of these operations are performed through an upper midline incision. However, a transverse incision has been reported to be superior to a midline incision in preventing postoperative intestinal obstruction. A transverse incision was also recommended to improve postoperative respiratory function, and to prevent incisional hernia [1]. However, few studies have compared these two methods in a prospective manner [2-4]. We performed a prospective randomized study to evaluate the differences between these two incisional methods for gastrectomy.

\section{Patients and methods}

Patients undergoing curative distal gastrectomy or total gastrectomy for gastric cancer between June 1994 and December 2003 at the Department of Surgery of Teikyo University Hospital were recruited for this study. This study protocol conformed to the ethical guidelines of our hospital and the Helsinki declaration. The patients were enrolled after informed consent had been obtained. Patients who had undergone any major laparotomy prior to gastrectomy were excluded.

The eligible patients were randomly allocated to have either an upper midline incision or a transverse incision. Upper midline incisions were generally performed from the xiphoid process of the sternum to approximately $2 \mathrm{~cm}$ below the umbilicus. Transverse incisions were performed at approximately $2 \mathrm{~cm}$ below the bilateral costal arches. The surgical procedures of gastrectomy, other than the method of laparotomy, were determined by the attending surgeons. Incisional length was also decided by the attending surgeons. A bioresorbable membrane to prevent adhesions was not used. Both 
types of incision were closed layer-to-layer in two layers. In most of the patients, the peritoneum and fascia were closed with a continuous 1-0 polydioxanone suture (PDS; Ethicon, Somerville, NJ, USA). Skin was closed with 2-0 or 3-0 silk interrupted stitches. The length of the incision was measured after surgery. The opening time was defined as the time from the start of the skin incision to the completion of the peritoneal incision. Closing time was defined as the time from the start of the inner suture to the completion of the skin suture. Staging of the disease was performed, using the TNM classification, after surgery. Peripheral venous blood was collected immediately after the operation and in the morning on postoperative day (POD) 1 , and the white blood cell (WBC) count and levels of C-reactive protein (CRP) and creatine phosphokinase (CK) were determined. The duration of continuous epidural analgesia after surgery was also determined by the attending surgeons. Administration of postoperative analgesics, in addition to continuous epidural analgesia, was decided by the nursing staff, who were not aware of the details of the study, according to the orders of the attending surgeons and as demanded by the patients. The occurrence of major postoperative complications during the perioperative period, including pneumonia and wound infection, was recorded by the attending surgeons. After discharge, all patients were followed up at our hospital, and the occurrence of intestinal obstruction necessitating readmission was recorded. The diagnosis of postoperative intestinal obstruction was made based on both physical examination and abdominal X-ray findings. Only those patients who had been followed for longer than 1 year after gastrectomy were included for the analysis of the incidence of postoperative intestinal obstruction. Cases of intestinal obstruction caused by the recurrence of malignancy were excluded. The study was terminated if the patient underwent another operation via laparotomy; otherwise, the study was terminated on the day of the latest consultation at the outpatient clinic of our hospital.

Data values are expressed as means \pm SE. Statistical analysis was performed with Student's $t$-test or the $\chi^{2}$ test. A difference was considered significant if the $P$ value was less than 0.05 .

\section{Results}

\section{Patient characteristics}

Distal gastrectomy. Two hundred and ninety-four patients who underwent distal gastrectomy were enrolled. However, 16 patients were excluded from the analysis, because the patients died after surgery while in hospital or were transferred to other hospitals from our hospital before discharge. Two other patients were revealed to have not fulfilled the study criteria after surgery and were also excluded. (A bioresorbable membrane was used in 1 patient. The other patient was revealed to have undergone major laparotomy.) Thus, 139 patients undergoing midline incision (group-DM) and 137 patients undergoing transverse incision (group-DT) were evaluated. There were no significant differences in age, sex, stage of the disease, and postoperative follow-up duration between group-DM and group-DT.

Total gastrectomy. One hundred and thirty-two patients who underwent total gastrectomy were enrolled. However, 13 patients were excluded from the analysis, because the patients died after surgery while in hospital or were transferred to other hospitals before discharge. Thus, 60 patients undergoing upper midline incision (group-TM) and 59 patients undergoing transverse incision (group-TT) remained for evaluation. There were no significant differences in age, sex, stage of the disease, and postoperative follow-up duration between group-TM and group-TT (Table 1).

\section{Operative details}

Distal gastrectomy. Times for both opening and closing the abdominal cavity were significantly longer in groupDT than in group-DM. The time taken for the surgical procedures for gastrectomy other than the opening and closing of the abdominal cavity was not different between these two groups. The total operative time was not different between the two groups. Incision length was significantly longer in group-DT than in group-DM. Operative blood loss was not significantly different between the two study groups.

Total gastrectomy. Times for both opening and closing of the abdominal cavity were significantly longer in group-TT than in group-TM. However, both the time for the surgical procedures other than opening and closing the abdominal cavity and total operative time were not significantly different between these two study groups. Incision length was longer in group-TT than in group-MT. Operative blood loss was not significantly different between the two study groups (Table 2).

\section{Laboratory data}

The WBC count on POD 1 was higher, and levels of CK both immediately after distal gastrectomy and on POD 1 were higher in group-DT than in group-DM. The levels of CK immediately after total gastrectomy and on POD 1 were higher in group-TT than in group-TM (Table 3). 
Table 1. Patient characteristics

\begin{tabular}{|c|c|c|c|c|}
\hline & \multicolumn{2}{|c|}{ Distal gastrectomy } & \multicolumn{2}{|c|}{ Total gastrectomy } \\
\hline & $\begin{array}{l}\text { Midline incision } \\
\quad(n=139)\end{array}$ & $\begin{array}{c}\text { Transverse incision } \\
\qquad(n=137)\end{array}$ & $\begin{array}{l}\text { Midline incision } \\
\quad(n=60)\end{array}$ & $\begin{array}{c}\text { Transverse incision } \\
(n=59)\end{array}$ \\
\hline Age (years) & $62.5 \pm 1.1$ & $63.4 \pm 1.0$ & $64.8 \pm 1.2$ & $63.0 \pm 1.2$ \\
\hline Sex (male:female) & $93: 46$ & $101: 36$ & $38: 22$ & $43: 16$ \\
\hline \multicolumn{5}{|l|}{ Stage of cancer } \\
\hline I & $89(64.0 \%)$ & $81(59.1 \%)$ & $12(20.0 \%)$ & $15(25.4 \%)$ \\
\hline II & $16(11.5 \%)$ & $21(15.3 \%)$ & $10(16.6 \%)$ & $8(13.6 \%)$ \\
\hline III & $21(15.1 \%)$ & $26(19.0 \%)$ & $15(25.0 \%)$ & $9(15.3 \%)$ \\
\hline IV & $13(9.4 \%)$ & $9(6.6 \%)$ & $23(38.3 \%)$ & $27(45.8 \%)$ \\
\hline Follow-up duration (days) & $1122 \pm 79$ & $1033 \pm 76$ & $725 \pm 106$ & $781 \pm 114$ \\
\hline
\end{tabular}

No significant difference was found between the two incisional methods in either the distal gastrectomy group or total gastrectomy group (Student's $t$-test or $\chi^{2}$ test)

Table 2. Operative details

\begin{tabular}{|c|c|c|c|c|}
\hline & \multicolumn{2}{|c|}{ Distal gastrectomy } & \multicolumn{2}{|c|}{ Total gastrectomy } \\
\hline & Midline incision & Transverse incision & Midline incision & Transverse incision \\
\hline \multicolumn{5}{|l|}{ Operative time (min) } \\
\hline Opening & $6.5 \pm 0.3$ & $10.0 \pm 0.3^{* *}$ & $6.6 \pm 0.6$ & $10.1 \pm 0.5^{* *}$ \\
\hline Closing & $17.0 \pm 0.6$ & $21.6 \pm 0.8^{* *}$ & $18.0 \pm 1.1$ & $21.8 \pm 1.0^{*}$ \\
\hline Other procedures & $173.3 \pm 4.6$ & $175.7 \pm 5.0$ & $265.8 \pm 10.8$ & $251.9 \pm 9.2$ \\
\hline Total & $196.4 \pm 4.7$ & $207.3 \pm 5.0$ & $290.5 \pm 11.1$ & $283.8 \pm 9.6$ \\
\hline Incision length $(\mathrm{cm})$ & $18.9 \pm 0.4$ & $21.1 \pm 0.5^{* *}$ & $22.5 \pm 0.8$ & $26.3 \pm 0.8^{* *}$ \\
\hline Intraoperative blood loss (ml) & $319 \pm 21$ & $329 \pm 27$ & $769 \pm 66$ & $980 \pm 172$ \\
\hline
\end{tabular}

$* P<0.05 ; * * P<0.01$ Versus midline incision for each operative method (Student's $t$-test)

Table 3. Laboratory data immediately after surgery (postoperative day [POD] 0) and on POD 1

\begin{tabular}{|c|c|c|c|c|}
\hline & \multicolumn{2}{|c|}{ Distal gastrectomy } & \multicolumn{2}{|c|}{ Total gastrectomy } \\
\hline & Midline incision & Transverse incision & Midline incision & Transverse incision \\
\hline \multicolumn{5}{|l|}{ POD 0} \\
\hline $\mathrm{WBC}\left(\times 10^{2} / \mathrm{mm}^{3}\right)$ & $107.7 \pm 2.9$ & $114.6 \pm 3.3$ & $113.9 \pm 5.2$ & $114.3 \pm 4.8$ \\
\hline $\mathrm{CRP}(\mathrm{mg} / \mathrm{dl})$ & $0.5 \pm 0.1$ & $0.4 \pm 0.1$ & $0.8 \pm 0.2$ & $0.7 \pm 0.1$ \\
\hline CK (IU) & $113 \pm 14$ & $249 \pm 13^{* *}$ & $142 \pm 13$ & $288 \pm 21 * *$ \\
\hline \multicolumn{5}{|l|}{ POD 1} \\
\hline WBC $\left(\times 10^{2} / \mathrm{mm}^{3}\right)$ & $106.5 \pm 2.1$ & $116.2 \pm 2.6^{*}$ & $122.4 \pm 5.0$ & $123.4 \pm 4.0$ \\
\hline $\mathrm{CRP}(\mathrm{mg} / \mathrm{dl})$ & $7.9 \pm 0.3$ & $8.2 \pm 0.3$ & $8.9 \pm 0.4$ & $10.2 \pm 0.6$ \\
\hline CK (IU) & $455 \pm 51$ & $1206 \pm 101 * *$ & $649 \pm 109$ & $1182 \pm 181 *$ \\
\hline
\end{tabular}

$* P<0.05 ; * * P<0.01$ Versus midline incision for each operative method (Student's $t$-test)

\section{Postoperative analgesia}

Distal gastrectomy. There was no significant difference in the number of doses of postoperative analgesics from the operative day to POD 3 between the two study groups when all patients were considered. However, in the patients in whom continuous epidural analgesia was used from the operative day until POD 2 or longer, the number of doses of additional postoperative analgesics was significantly smaller in group-DT than in groupDM.

Total gastrectomy. There was no significant difference in the number of doses of postoperative analgesics from the operative day until POD 3 between the two study groups, even in patients receiving continuous epidural analgesia (Table 4). 
Table 4. Number of doses of postoperative analgesics

\begin{tabular}{|c|c|c|c|c|}
\hline & \multicolumn{2}{|c|}{ Distal gastrectomy } & \multicolumn{2}{|c|}{ Total gastrectomy } \\
\hline & Midline incision & Transverse incision & Midline incision & Transverse incision \\
\hline All patients & $\begin{aligned} 3.3 & \pm 0.2 \\
(n & =139)\end{aligned}$ & $\begin{aligned} 2.9 & \pm 0.2 \\
(n & =137)\end{aligned}$ & $\begin{aligned} 4.0 & \pm 0.4 \\
(n & =60)\end{aligned}$ & $\begin{aligned} 3.4 & \pm 0.3 \\
(n & =59)\end{aligned}$ \\
\hline Patients with epidural analgesia & $\begin{aligned} 3.3 & \pm 0.2 \\
(n & =100)\end{aligned}$ & $\begin{aligned} 2.6 & \pm 0.2^{*} \\
(n & =115)\end{aligned}$ & $\begin{aligned} 3.7 & \pm 0.4 \\
(n & =48)\end{aligned}$ & $\begin{aligned} 3.1 & \pm 0.3 \\
(n & =50)\end{aligned}$ \\
\hline
\end{tabular}

$* P<0.05$ Versus midline incision in the distal gastrectomy group (Student's $t$-test)

Number of doses of analgesics, excluding continuous epidural analgesia, from the operation day to postoperative day 3

Table 5. Postoperative complications

\begin{tabular}{|c|c|c|c|c|}
\hline & \multicolumn{2}{|c|}{ Distal gastrectomy } & \multicolumn{2}{|c|}{ Total gastrectomy } \\
\hline & Midline incision & Transverse incision & Midline incision & Transverse incision \\
\hline \multicolumn{5}{|l|}{ Perioperative period } \\
\hline Wound infection & $7 / 139(5.0 \%)$ & $7 / 137(5.1 \%)$ & $1 / 60(1.7 \%)$ & $5 / 59(8.5 \%)$ \\
\hline Pneumonia & $9 / 139(6.5 \%)$ & $2 / 137(1.5 \%)^{*}$ & $2 / 60(3.3 \%)$ & $4 / 59(6.8 \%)$ \\
\hline Total major complications & $26 / 139(18.7 \%)$ & $19 / 137(13.9 \%)$ & $16 / 60(26.7 \%)$ & $20 / 59(33.9 \%)$ \\
\hline \multicolumn{5}{|l|}{ After discharge } \\
\hline \multicolumn{5}{|l|}{ Intestinal obstruction } \\
\hline All patients & $12 / 139(8.6 \%)$ & $3 / 137(2.2 \%)^{*}$ & $7 / 60(11.7 \%)$ & $3 / 59(5.1 \%)$ \\
\hline Stage I-III & $9 / 126(7.1 \%)$ & $0 / 128(0.0 \%)^{* *}$ & $4 / 37(10.8 \%)$ & $3 / 32(9.4 \%)$ \\
\hline
\end{tabular}

$* P<0.05 ; * * P<0.01$ Versus midline incision $\left(\chi^{2}\right.$ test $)$

Number of patients with complication/total number of patients

\section{Postoperative complications}

Distal gastrectomy. The incidence of postoperative pneumonia was higher in group-DM than in group-DT. The incidences of wound infection and of total postoperative major complications were almost equal in the two groups. Wound dehiscence occurred in only one patient, in group-DM. There was no case of incisional hernia. The incidence of postoperative intestinal obstruction requiring readmission to hospital after discharge was significantly lower in group-DT than in group-DM. Of the patients with stage I-III cancer, snone developed postoperative intestinal obstruction in group-DT.

Total gastrectomy. The incidence of wound infection, pneumonia, and total postoperative complications was not different between the two groups. There was no case of wound dehiscence or incisional hernia in either group. In contrast to the distal gastrectomy patients, no difference was found in the incidence of postoperative intestinal obstruction between the two groups after total gastrectomy, even in the patients with early-stage cancer (Table 5).

\section{Discussion}

In the present study we compared midline incision with transverse incision for gastrectomy. For both total and distal gastrectomies a transverse incision needed a longer time to open and close the abdominal cavity than a midline incision; however, after distal gastrectomy, the number of doses of postoperative analgesics was smaller in the transverse-incision group than in the midline-incision group in those patients with continuous epidural anesthesia. The incidence of postoperative pneumonia and postoperative intestinal obstruction was lower in the transverse incision group after distal gastrectomy. However, these beneficial effects of transverse incision were not observed after total gastrectomy.

Both the opening time and the closing time of the incision were longer in the transverse-incision group than in the upper midline-incision group in our study. The WBC count and plasma CK level were higher in the transverse-incision group than in the midline-incision group. These results suggest that surgical stress or muscle damage may be more severe with a transverse incision than with a midline incision. However, total operative time was not different between the two incisional methods. Transverse incision did not increase operative blood loss or postoperative complications. 
Thus, we believe that the more severe stress of a transverse incision can be ignored in practice.

Halasz [2] reported that both the analgesic requirement and pulmonary complications after transverse incisions were less than those after vertical incisions in his prospective study. In contrast, Greenall et al. [5,6] reported that the direction of the incision did not have a significant effect on postoperative pulmonary complications. However, in their study, all patients undergoing any major laparotomy were included, and they did not use epidural analgesia. It was also reported that intercostal nerve block was more effective after subcostal incisions than after midline incisions, for pain relief and improvement in pulmonary function [7]. In our study, we showed that a transverse abdominal incision was superior to an upper midline incision for decreasing pneumonia after distal gastrectomy and for attenuating wound pain in patients receiving epidural analgesia after distal gastrectomy. A transverse incision may be more beneficial for pain relief and pulmonary function during the immediate postoperative days than a midline incision, especially in patients receiving continuous epidural analgesia postoperatively.

The effects of attenuation of wound pain and decreasing the incidence of pneumonia in patients receiving epidural analgesia were not observed after total gastrectomy. The reason for the difference between the surgical procedures was not clear from this study; however, the complicated operative procedure and longer incision length with total gastrectomy may mask these beneficial effects of transverse incision.

A transverse abdominal incision is expected to be superior to a midline incision for preventing postoperative intestinal obstruction, because adhesion between the abdominal wall and intestinal tract is less marked in patients with a transverse incision than in those with a midline incision. It was reported that the incidence of postoperative intestinal obstruction did not differ between the two incisional methods [8]. However, that study was performed in a retrospective manner and the number of patients who underwent transverse incision was small. In our study, the comparison was performed in a prospective randomized manner. The incidence of postoperative intestinal obstruction was lower after transverse incision than after midline incision in patients who underwent distal gastrectomy in our study. This result supports the hypothesis that a transverse abdominal incision may be superior to an upper midline incision for preventing postoperative intestinal obstruction.

There was no significant difference in the incidence of postoperative intestinal obstruction between the two incisional methods in patients with total gastrectomy.
The reasons were not clear from our data; however, three possible reasons can be advanced. Firstly, the incidence of advanced-stage cancer was higher in patients with total gastrectomy than in those with distal gastrectomy. Thus, it is possible that some of the cases of intestinal obstruction may have been caused by cancer recurrence, although, as far as possible, we excluded patients in whom the obstruction was caused by cancer. Secondly, adhesion other than that between the abdominal wall and intestinal tract may cause intestinal obstruction after total gastrectomy, because the operative procedure of total gastrectomy is much more complicated than that of distal gastrectomy. It was reported that the incidence of postoperative intestinal obstruction after gastrectomy proved to be lower in patients with minimally invasive surgery [9]. The present report supports the second possibility above. Thirdly, the number of patients who underwent total gastrectomy was much smaller than the number of patients who underwent distal gastrectomy. Further investigation is needed to evaluate the difference in the two incisional methods for total gastrectomy.

In conclusion, for distal gastrectomy, a transverse abdominal incision may be more beneficial than an upper midline incision, to attenuate postoperative wound pain, decrease the incidence of postoperative pneumonia, and prevent postoperative intestinal obstruction.

\section{References}

1. Blomstedt B, Welin-Berger T. Incisional hernias. Acta Chir Scand 1972;138:275-8.

2. Halasz NA. Vertical vs horizontal laparotomies. Arch Surg 1964; 88:911-4.

3. Stone HH, Hoefling SJ, Strom PR, Dunlop WE, Fabian TC. Abdominal incisions: transverse vs vertical placement and continuous vs interrupted closure. South Med J 1983;76:1106-12.

4. Haga M, Otani N, Kiyokawa K, Kawakami T. Pararectal versus transverse incision for retroperitoneal approach to aorto-iliac region (in Japanese with English abstract). Jpn J Cardiovasc Surg 1998;27:293-6.

5. Greenall MJ, Evans M, Pollock AV. Midline or transverse laparotomy? A random controlled clinical trial. Part I. Influence on healing. Br J Surg 1980;67:188-90.

6. Greenall MJ, Evans M, Pollock AV. Midline or transverse laparotomy? A random controlled clinical trial. Part II. Influence on postoperative pulmonary complications. Br J Surg 1980;67:191-4.

7. Engbreg G. Single-dose intercostal nerve blocks with etidocaine for pain relief after upper abdominal surgery. Acta Anaesthesiol Scand 1975;60:43-9.

8. Shimizu K, Yoshida K, Hirai T, Toge T. Relationship between surgical approach and postoperative ileus in gastric cancer (in Japanese with English abstract). Jpn J Clin Surg 2003;64:801-4.

9. Nashimoto A, Morota T, Yabusaki H, Tsuchiya Y, Tanaka O, Sasaki J. Investigation of postoperative ileus after gastrectomy and prevention of ileus by limited surgery for early gastric cancer (in Japanese with English abstract). Jpn J Gastroenterol Surg 2000; 33:1455-60. 